\title{
Christiania, Science und Cocktails
}

\author{
Soziale Experimente in Dänemark
}

\author{
Korrespondenzadresse \\ Prof. Dr. Dr. Manfred Spitzer \\ Universität Ulm \\ Abteilung für Psychiatrie \\ Leimgrubenweg 12-14 \\ 87054 Ulm
}

Bibliografie

DOI https://doi.org/10.1055/a-0883-3323

Nervenheilkunde 2019; 38: 453-458

(c) Georg Thieme Verlag KG Stuttgart · New York

ISSN 0722-1541
Als mich im Januar 2019 eine E-Mail aus Dänemark samt Einladung zu einem Vortrag in Kopenhagen erreichte, war ich mir zunächst nicht sicher. Der Absender war Science and Cocktails, eine Gruppe junger Leute, die seit Jahren eine Art Vorlesungsreihe gleichen Namens in Kopenhagen organisieren. Der Ort war bis vor kurzem ein altes Kino und ist mittlerweile eine alte Halle in der im Herzen von Kopenhagen gelegenen „dänischen Freistadt“ Christiana. Diese Gemeinde war mir bislang unbekannt, und weil das Ganze ziemlich schräg klang, beantwortete ich die Einladung aus purer Neugier zaghaft positiv. Wenige E-Mails später war die Sache eingetütet und Mitte Mai 2019 ging es dann für ein Wochenende in das kleine nordeuropäische Land, dessen einzige Landgrenze die mit Deutschland ist und das 5,7 Millionen Einwohner hat. Und einmal mehr galt die alte ostchinesische Weisheit: Wenn einer einen Vortrag hält, dann lernt er selbst am meisten - über eine unmögliche aber dennoch existierende Stadt, Kommunikation in der Wissenschaft und vor allem über Dänemark und die Dänen.

Obwohl es gerade im Winter wenig Sonne und viel trübe Regentage gibt, sind die Dänen das glücklichste Völkchen der Erde. Wer denkt, dass das vielleicht ein Zufall sei und dass die Dänen doch letztlich ganz ähnlich lebten wie wir auch, der irrt. Statt nun Unterschiede aufzulisten, möchte ich sie an einem Beispiel illustrieren. Das Beispiel heißt Christiania.

\section{Christiania}

Wenn hierzulande vor Jahrzehnten einzelne Häuser in Städten, oder vor einem Jahr ein paar Dutzend Baumhäuser in einem kleinen Wäldchen besetzt werden, dann schreitet irgendwann die Polizei mit all ihrer "Staatsgewalt“ ein und wirft die Leute heraus. ${ }^{1}$

1 In Wikipedia findet man zum Hambacher Forst die folgenden Angaben: Bei den Großdemonstrationen im Herbst 2018 ging es um die Räumung von seit 2012 bestehenden Baumhäusern und die für Oktober geplante Abholzung von ca. 100 Hektar, angeblich wegen fehlendem Brandschutz. Faktisch ging es jedoch um die Fortführung des Braunkohletagebaus durch den Energieversorger RWE. „Nachdem die Rodung durch das Oberverwaltungsgericht Münster vorerst gestoppt wurde und eine Großdemonstration am Wald mit ca. 50000 Teilnehmern stattfand, stellte die Landesregierung die Räumung ein. Nach Schätzungen der Gewerkschaft der Polizei leistete die Polizei insgesamt rund
Als am 4. September des Jahres 1971 Einwohner der umliegenden Stadtteile ein mitten in Kopenhagen gelegenes verlassenes Militärgelände von 34 Hektar Größe besetzten, geschah hingegen erst einmal - nichts! Man sagt heute gerne, es seien „Hippies“ gewesen, wiewohl diese Bezeichnung nicht wirklich klar macht, wen genau man damit meint. Sehr wahrscheinlich traf dies zu Beginn nicht, und allenfalls im Laufe der weiteren Entwicklung teilweise, zu. Das Gelände wurde zunächst als Kinderspielplatz genutzt und dann als Wohnraum für Obdachlose und andere Menschen, die günstigen Wohnraum brauchten. Denn der ist in Kopenhagen Mangelware. Die Übernahme war nicht organisiert und erfolgte auch aus Protest gegen die dänische Regierung und deren Unfähigkeit, für bezahlbaren Wohnraum zu sorgen.

Ende September 1971 publizierte der Journalist und (wie man heute sagen würde) Aktivist Jacob Ludvigsen in einer von ihm selbst herausgegebenen Zeitung für junge Menschen einen Artikel, in dem er die „verbotene Stadt des Militärs“ für eröffnet erklärt und zugleich weit bekannt gemacht hatte. Danach entwickelte sich das besetzte Gelände schnell in Richtung Kollektivismus und Anarchismus - in starkem Kontrast zur vorherigen Nutzung durch das Militär ( Abb. 1, $>$ Abb. 2).

„Christiania ist das Land der Siedler. Es ist bis jetzt die größte Chance, eine Gesellschaft von Null aufzubauen - und dabei trotzdem die vorhandenen Gebäude weiter zu nutzen. Ein eigenes Elektrizitätswerk, ein Badehaus, eine gigantische Sporthalle, wo all die Friedenssuchenden würdevoll meditieren können, und ein Yogazentrum. Hallen, in denen sich Theatergruppen zu Hause fühlen können. Gebäude für Kiffer, die zu paranoid und schwach sind, um sich abzuhetzen“, schrieb Ludvigsen in seinem Artikel. Das damals ebenfalls von ihm mitverfasste Leitbild für das neue Zusammenleben lautete:

„Das Ziel von Christiania ist das Erschaffen einer selbst-regierenden Gesellschaft, in der alle und jeder für sich für das Wohlergehen der gesamten Gemeinschaft verantwortlich ist. Unsere Gesellschaft soll ökonomisch selbsttragend sein, und als solche ist es unser Be-

eine Million Arbeitsstunden in den 5 Wochen der Räumung. "(Die angegebene Quelle, der Bonner Generalanzeiger vom 8.10.2018, erscheint seriös.) 


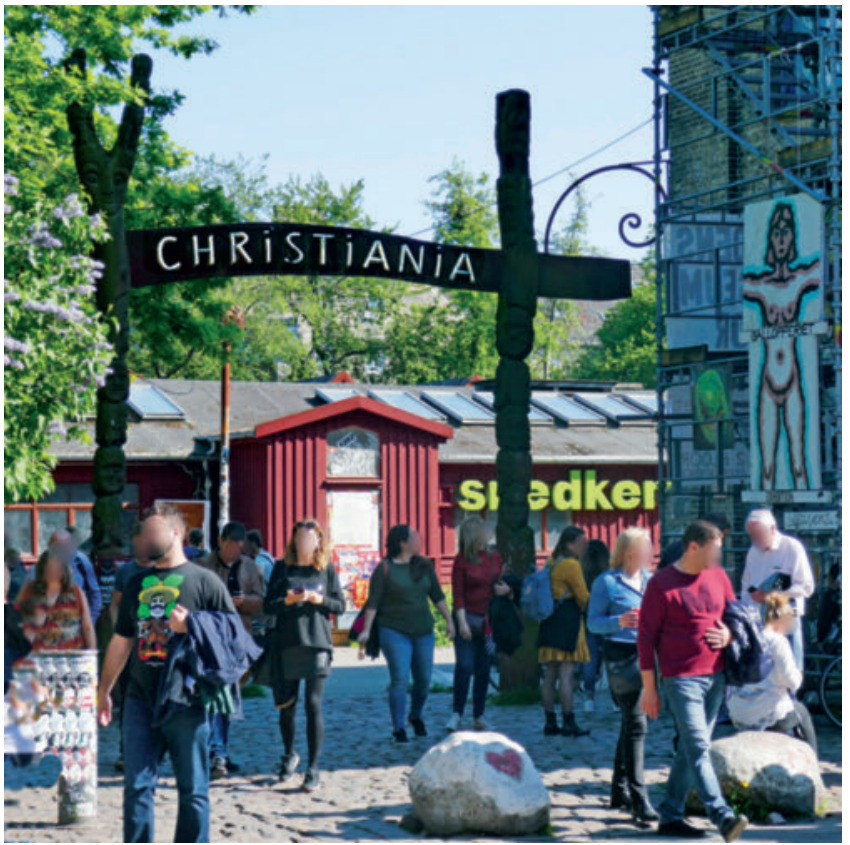

- Abb. 1 Der Eingang der „Freistadt Christiania“ ist mittlerweile weltbekannt. (Quelle: @Autor)

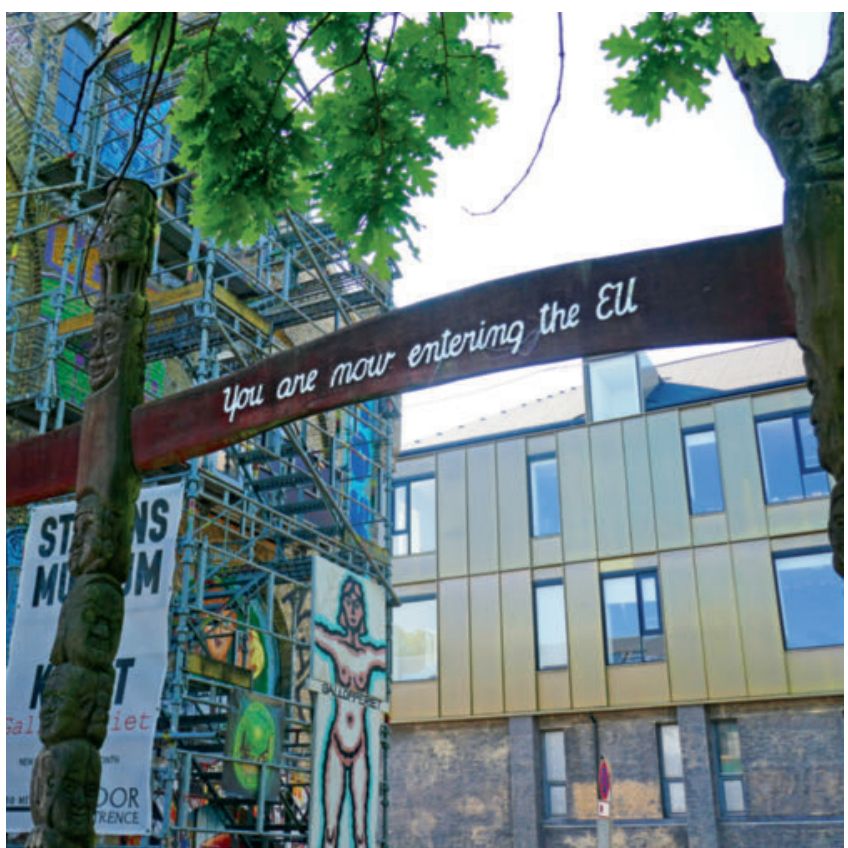

- Abb.2 Die Rückseite des Eingangsschildes macht deutlich, dass man die Freistadt wieder verlässt und die EU betritt. Auch die Lage der Christiania inmitten unspektakulärer städtischer Bebauung wird deutlich. (Quelle: @Autor)

streben, unerschütterlich in unserer Überzeugung zu sein, dass psychologische und physische Armut verhindert werden kann."

Über Jahrzehnte war unklar, was geschehen sollte. Mehrere Versuche der Räumung scheiterten am Widerstand der einigen hundert Bewohner. Gemäß einer Übereinkunft mit dem dänischen Verteidigungsministerium bezahlen sie seit dem Jahr 1995 Steu-

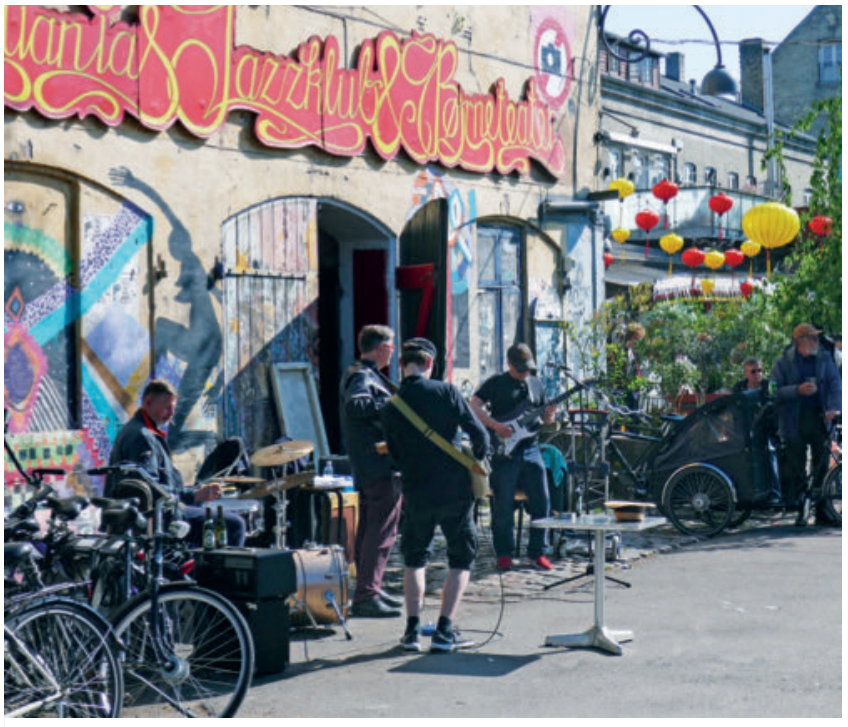

- Abb. 3 Straßenecke zur Pusher-Street im Hintergrund. An der Hauswand im Hintergrund das Zeichen für „Fotografieren verboten “. Darunter die gelben und roten Lampions, die anzeigen, dass weiche Drogen verkauft werden. In den Seitenstraßen geht es hingegen ganz normal zu, mit Musik (eine ältere Rock-Band), kleinen Läden, Essen und (vor allem Bier) Trinken. (Quelle: @Autor)

ern sowie Gebühren für Wasser, Strom und Müllentsorgung. In einem Teilgebiet der Christiania, der Pusher Street („Drogendealer Straße“), herrscht strenges Fotografieverbot ( $\boldsymbol{A}$ Abb. 3). Denn dort wird an vielen Buden Cannabis in den verschiedensten Formen und Sorten verkauft - mehr oder weniger geduldet von der dänischen Polizei, die mal zuschaut und mal Razzien durchführt. Ansonsten fühlt sich die Christiania eher an wie eine innerstädtische Freizeitanlage ( $\triangleright$ Abb. 4$)$ mit eingegliedertem Wochenmarkt.

Bis heute taten die dänischen Behörden insgesamt recht wenig. Aus Sicht der dänischen Behörden ist die Christiania also so etwas wie eine staatlich geduldete autonome Gemeinde. Mit anderen Worten: Mitten in Dänemarks Hauptstadt gibt es seit fast einem halben Jahrhundert (!) einen nahezu rechtsfreien Raum, dessen Geschicke von Autonomen, Hausbesetzern, Hippies, Drogensüchtigen und Rockerbanden (die den Drogenhandel unter sich aufteilen) bestimmt werden. Den Marktwert der 34 Hektar in der besten Lage der Hauptstadt möchte man sich gar nicht vorstellen; die Konflikte, welche von einer wöchentlich tagenden Versammlung der Bewohner zu bewältigen sind, auch nicht. ${ }^{2}$ Denn auch eine vermeintliche Anarchie kommt nicht ohne Regeln aus. Diese Regeln erlauben u. a. den Handel mit und den Konsum weicher Drogen (Cannabis), und sie verbieten Diebstahl, Gewalt, harte Drogen, Feuerwaffen, gefährliche Messer, kugelsichere Westen (kein Witz!) und die Abzeichen von Motorradclubs wie „Hells Angels“.

Aber was heißt hier eigentlich „Marktwert"? Mittlerweile gehört die Christiania zu den größten Touristenattraktionen Kopen-

2 Das Plenum (etwa 400 Menschen) behandelt die Gemeinschaft betreffende Angelegenheiten, beschließt also beispielsweise den jährlichen Haushaltsplan und führt Verhandlungen mit dem dänischen Staat und mit der Kopenhagener Polizei. 


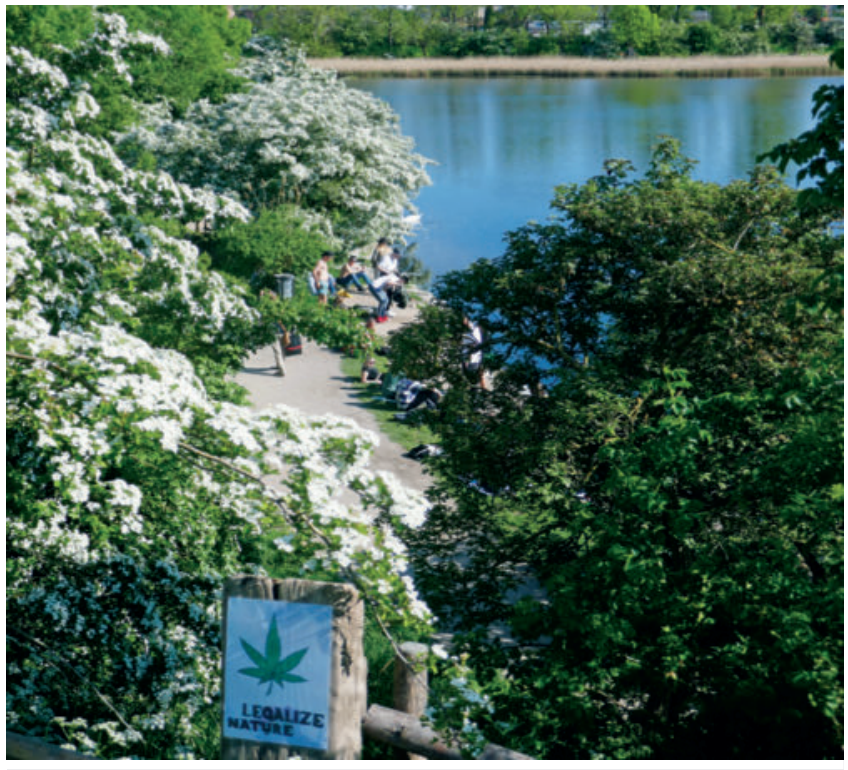

- Abb.4 Nur wenige Meter von den Verkaufsständen entfernt wird es ruhig und die Christiania fühlt sich - fast (vgl. das Hinweisschild im Vordergrund) - an wie eine Parkanlage. (Quelle: @Autor)

hagens. Sie wird von vielen Dänen als Symbol und Inbegriff für den eigenen freien Lebensstil gesehen, auch von Institutionen, Organisationen und Unternehmen: Wer seinen ausländischen Gästen etwas „typisch Dänisches“ bieten will, der geht mit ihnen in die Christiania! - Nicht anders erging es mir ja auch. Und mein Gastgeber, ein deutscher Student der Astrophysik, der seit 2 Jahren dort lebt, zeigte und erklärte mir die Christiania mit Stolz und Begeisterung, was ich beim Anblick der sehr vielen Menschen, die beim Bier oder einer Rauchware (was auch immer) nahezu wie auf einem Jahrmarkt sich unterhielten, gut nachvollziehen konnte. Hier wird seit einem knappen halben Jahrhundert ein soziales Experiment gelebt!

\section{Science and Cocktails}

Wissenschaft gilt vielen Menschen als langweilig und vor allem „trocken" im Vergleich zum ansonsten vielleicht eher feucht-fröhlichen wirklichen Leben. Um diesen Gedanken etwas aufzuweichen und Wissenschaft „ins wirkliche Leben“ zu übertragen, gibt es die Veranstaltungsreihe Science and Cocktails. Die Grundidee wird auf ihrer Webseite wie folgt beschrieben:

„Science \& Cocktails ist eine Initiative, die Wissenschaft und Unterhaltung durch die Schaffung einer Reihe von öffentlichen Vorlesungen inmitten von Musik, Kunst und einem nebligen, mit Trockeneis gekühlten Cocktail in der Hand einander näherbringen wollen. Im Allgemeinen werden wissenschaftliche Erkenntnisse für die meisten Menschen als unzugänglich betrachtet, sodass sie wie jede andere Information auch vom Forscher zum Journalisten und weiter über die Kanäle der Medien verbreitet und dabei vielleicht etwas verdreht werden. [...] Nach unserer Überzeugung fehlt im städtischen Raum eine Nachtkultur, die sich aus Wissen, Diskussion und Unterhaltung entwickelt. Wir betrachten den wissenschaftlichen Ansatz nicht allein als Informationsgewinn, sondern auch als Inspiration für Musik und Kunst und als ein Werkzeug beim Mixen

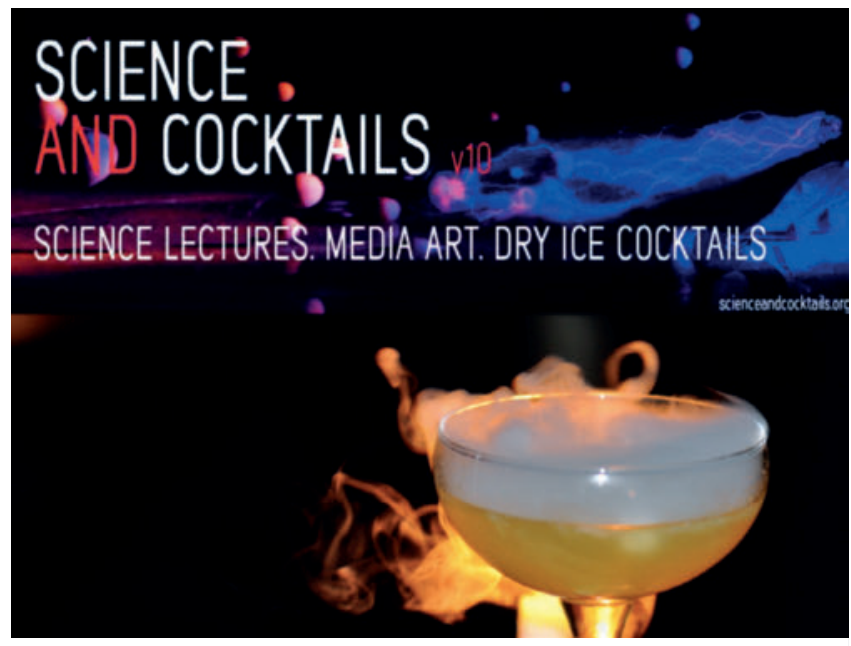

Abb. 5 Abbildungen, die man auf der Webseite von Science \& Cocktails findet, und deren grafische Gestaltung dessen Grundgedanken vielleicht besser verdeutlichen können als viele Worte (http://www.scienceandcocktails.org/). Nebenbei bemerkt: Trockeneis ist nichts anderes als sehr kaltes (tiefer als $-78,48^{\circ} \mathrm{C}$ gekühltes) Kohlendioxid (CO2), das bei normalem Luftdruck bei $-78,48^{\circ} \mathrm{C}$ direkt vom Feststoff in die Gasphase übergeht, also ohne vorher zu schmelzen. Wirft man ein etwa Würfelzucker-großes Stückchen in eine wässrige Flüssigkeit (Cocktail), sprudelt sehr viel kaltes, mit Wasserdampf gesättigtes Gas, und es entsteht Nebel (Quelle: http://www.scienceandcocktails.org).

von Cocktails, wodurch wir eine einzigartige Atmosphäre schaffen möchten “3 - für kreative Gedanken jeglicher Art, möchte man ergänzen.

Dies ist aus meiner Sicht ein bedeutender Schritt weg vom Dasein „im Elfenbeinturm“ hin zu dem, was Wissenschaft schon immer war und auch sein sollte: Das Streben neugieriger Menschen nach Wahrheit und Verständnis der Welt und ihrer selbst durch (systematische) Beobachtung und Austausch der gemachten Erfahrungen und damit verbundenen Gedanken. Trocken ist am gesamten Konzept also lediglich das Eis in den Cocktails ( A Abb.5).

Science and Cocktails begann im Jahr 2010 als ein Gemeinschaftsprojekt des Niels Bohr Instituts für Physik an der Universität von Kopenhagen und des Physikers Jacome (Jay) Armas ( $\mathbf{A} \mathbf{A b} \mathbf{b} . \mathbf{6}$ ). Seit 2011 finden die Veranstaltungen in der Christiania statt, was dem Ganzen eine gewisse Färbung im Sinne eines weiteren sozialen

3 Im Original und etwas ausführlicher: „Science \& Cocktails is an initiative that brings science and entertainment closer together by creating a series of public lectures intertwined with music/art performances and smoky dry-ice chilled cocktails in your hand. Scientific knowledge is in general not considered accessible by many and, like any other form of information, it travels from researcher to journalist and is finally transmitted through the usual media channels (but perhaps slightly distorted). Science \& Cocktails aims to create a relaxed platform where researchers and audience are on equal footing and conversations can be easily followed while sipping a Famous Penicillin cocktail side by side with your favorite scientist. It is our belief that a night culture that evolves around knowledge, discussion and entertainment is altogether lacking in urban spaces. Taking a scientific approach that extends not only through information flow but also as an inspiration in music/art and as a tool in the craft of cocktails we seek to create a unique atmosphere. " 


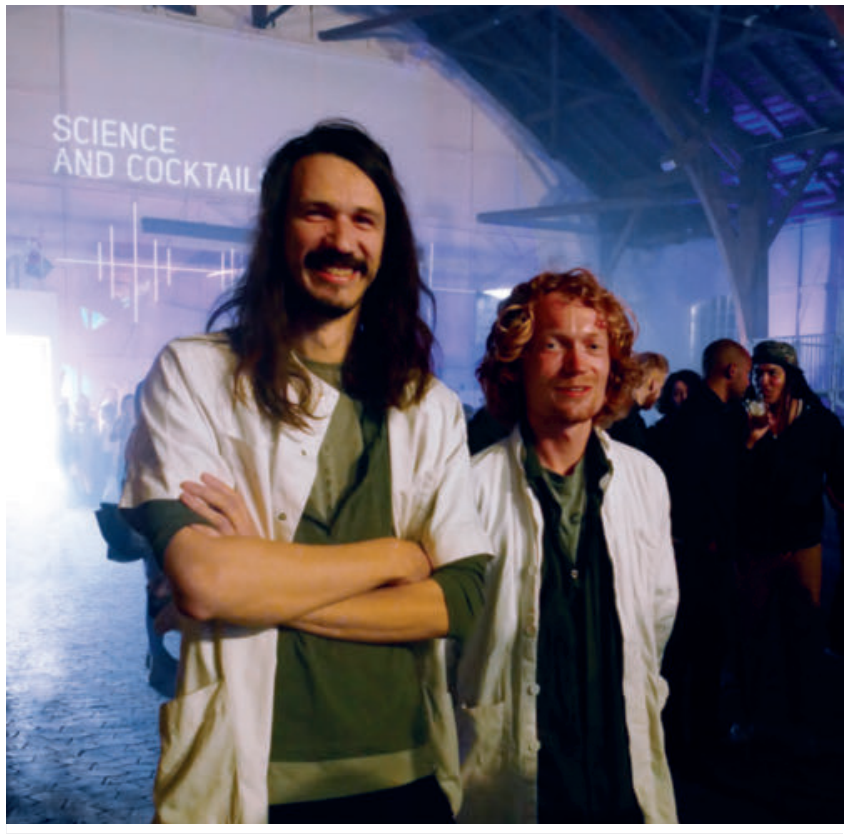

Abb. 6 Initiator und Organisator von Science \& Cocktails, Jay (links) und Phillip , mein unmittelbarer Ansprechpartner, aufmerksamer Gastgeber und kundiger Guide. Beide tragen, wie alle anderen Mitarbeiter des Teams an diesem Abend, weiße Kittel. (Quelle: @Autor)

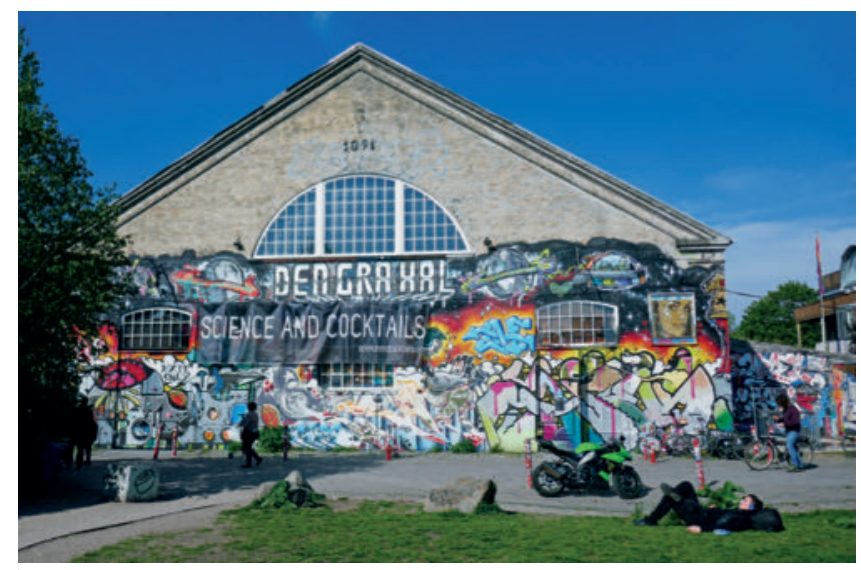

- Abb. 7 Die „graue Halle“, der große Veranstaltungsraum der Christiania mit Platz für über 1000 Besucher, ist mittlerweile längst nicht mehr ganz so grau wie früher. (Quelle: $\odot$ Autor)

Experiments gegeben hat und gibt. Als Vortragender wurde ich von Philipp Weber, einem Studenten der Astrophysik abgeholt, der zusammen mit weiteren jungen Leuten bei dem Projekt unentgeltlich mitarbeitet und mir sowohl „Science \& Cocktails“ als auch „Christiania“ mit viel Engagement und Sachkunde erklärte.

Seit 2014 ist Science \& Cocktails eine eingetragene Non-Profit-Organisation mit dem Ziel, die Kommunikation in der Wissenschaft zu verbessern. Mittlerweile gibt es entsprechende Veranstaltungen gleichen Namens auch in Brüssel (seit 2017), Amsterdam (seit 2018) und Johannesburg (seit 2015). Es ist also eine Art „Bewegung" entstanden, die mehrfach preisgekrönt wurde.

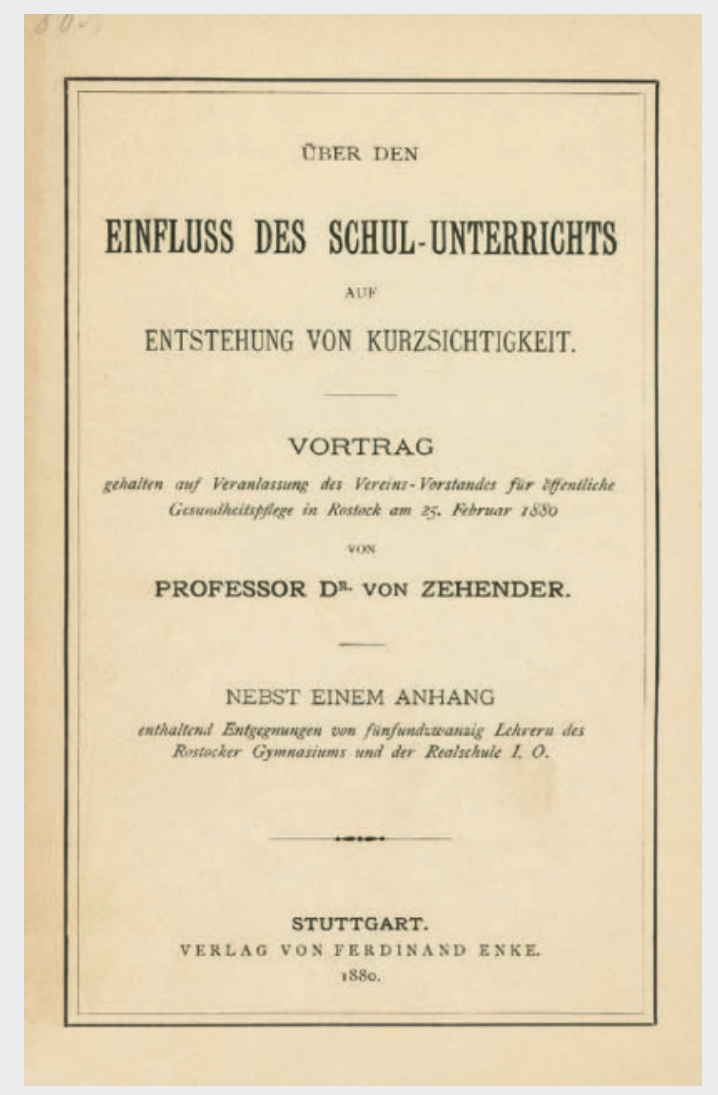

- Abb. 8 Titelseite der Publikation von Carl Wilhelm von Zehender. Bei der Lektüre des Anhangs kann man nachvollziehen, dass schon vor 140 Jahren das Verhältnis von Ärzten und Lehrern von gegenseitigem Misstrauen geprägt war (aus [5]).

\section{Digitales Dänemark}

Mein Vortrag am 18.5.2019 um 20 Uhr in der „grauen Halle“ ( $\triangleright$ Abb. 7) hatte den Titel The Smartphone Epidemic, ${ }^{4}$ und so redete ich in englischer Sprache, die in Dänemark wie im Norden Europas üblich jeder sehr gut beherrscht. Es ging um die Risiken und Nebenwirkungen des erstens weltweit mit etwa 5 Milliarden Nutzern am meisten verbreiteten, zweitens mit täglich je nach Studie und Land etwa 3 bis 5 Stunden am meisten genutzten, und drittens kleinsten digitalen Endgeräts. Ich begann damit, dass allein diese 3 Fakten zum Smartphone sich auf die Entwicklung der Augen weltweit verheerend auswirken. Denn die Augen wachsen so lange, bis die Geometrie stimmt und das Bild auf der Netzhaut scharf ist. Schaut man während der ersten 25 Jahre seines Lebens - der Zeit des möglichen Augenwachstums - für zu viele Stunden in die Nähe, schneiden sich die Strahlenweiter weiter hinten, weswegen das Auge zu lang wird [4]. Früher sprach man daher auch von Schulmyopie, und die erste Beschreibung des Phänomens findet sich in einer Publikation ( $\triangleright$ Abb. 8) des Rostocker Ordinarius für Ophthalmologie, Prof. Dr. Carl Wilhelm von Zehender, aus dem Jahr $1880 .{ }^{5}$

4 http://www.scienceandcocktails.org/cph/2019/SmartPhone.html

5 Für den Hinweis und die pdf-Datei bin ich Herrn Prof. Dr. Lothar Spillmann, Freiburg, herzlich dankbar. 


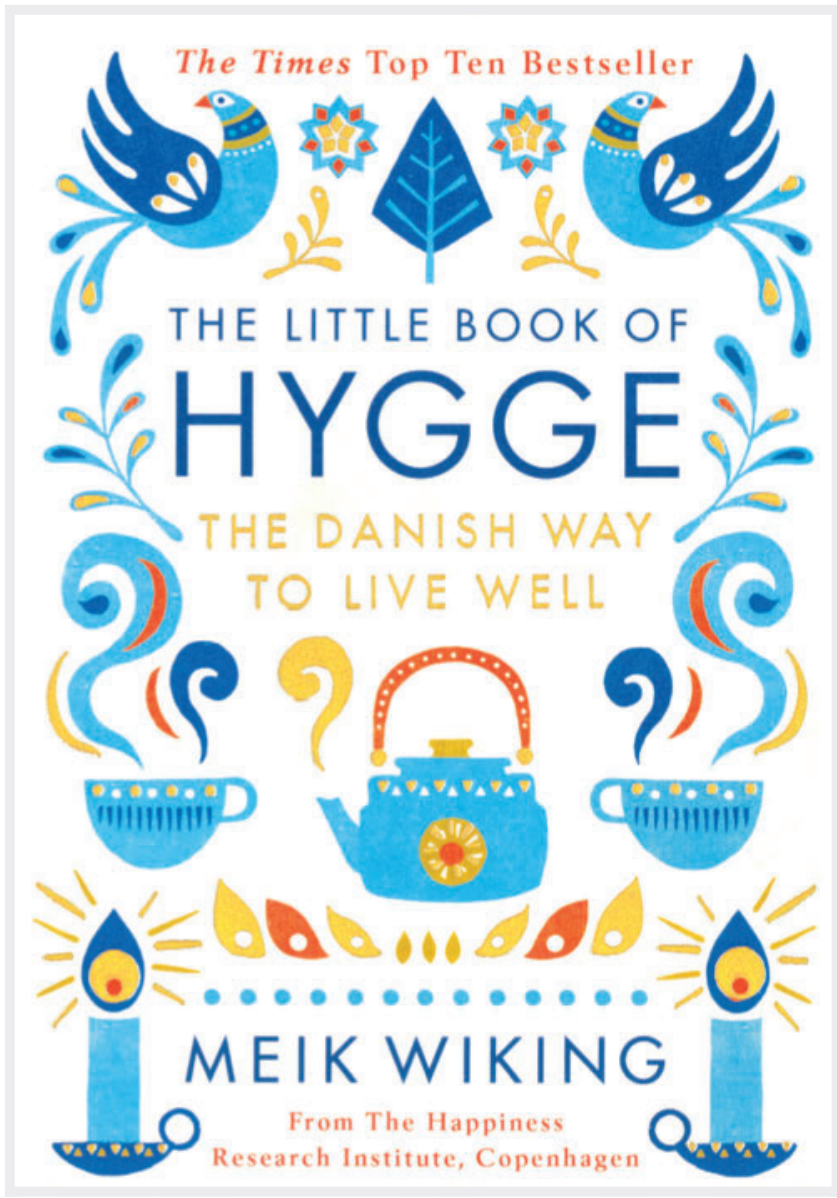

- Abb. 9 Im New York Times Bestseller „The little book of Hygge“, das kein anderer als der Chef des Happiness Research Institute in Kopenhagen, Meik Wiking, geschrieben hat, erfährt man viel zu diesem vielschichtigen Phänomen.
Damals gab es noch keine Smartphones, ebenso wenig beim Beginn des Schulwesens in Süd-Korea in den 1950er- und 1960er-Jahren, aber Kurzsichtigkeit durch Beschulung (und zu häufiges sehen in die Nähe) gab und gibt es bis heute. Weil nun das Smartphone gegenwärtig weltweit von sehr vielen Menschen sehr viel genutzt wird, kann man mittlerweile jedoch mit Fug und Recht von einer Pandemie der Kurzsichtigkeit, verursacht durch das Smartphone, sprechen. Denn das Phänomen besteht weltweit, mit Prävalenzraten bei der jungen Bevölkerung von 30-50\% (Europa), 80 \% (China) bis $95 \%$ (Südkorea).

Das dänische Publikum hatte davon offenbar noch nie etwas gehört. Auch nicht von Smartphone-Sucht oder der Tatsache, dass „Geosocial-networking Apps“ (tinder, grindr) zu millionenfachem Gelegenheitssex zwischen fremden Menschen und damit weltweit zu einem Anstieg von Geschlechtskrankheiten geführt haben. Im Gegenteil: Als ich dann noch erklärte, dass es sich bei etwa einem Drittel der im Internet bewegten Daten um Pornografie handelt, reagierte das Publikum deutlich spürbar abweisend. „Manfred, pass auf, dass du hier heil herauskommst, " schoss es mir plötzlich durch den Kopf - eine für mich neue Vortrags-Erfahrung. - Was tun? Beschwichtigen: „alles nicht so schlimm“? - „Auf keinen Fall!“ Ich hielt daraufhin den am wenigsten unterhaltsamen, faktenreichsten und kürzest möglichen Vortrag, den ich zu diesem Thema gehalten habe: Aufmerksamkeitsstörungen, Einsamkeit, Empathieverlust, Depression (einschließlich Suizid), Demenz, Diabetes, Bluthochdruck (und damit Schlaganfälle und Herzinfarkte), Haltungsschäden, Übergewicht, Ängste, Lebensunzufriedenheit, geringere Bildung, geringeres gegenseitiges Vertrauen, deutlich geringere Bildung, Fake-News, Radikalisierung, Manipulation, Verlust von Privatheit, Demokratiegefährdung und gesellschaftlicher Zerfall. Schließlich haben Smartphones den Alkohol als Ursache von Verkehrsunfällen bei der jungen Generation abgelöst. Das Ausmaß des durch ihren Gebrauch verursachten milliardenfachen Leids und Siechtums, die (leicht zu berechnenden) Millionen von zusätzlichen 
Toten (die Auswirkungen von Rauchen, Tabaklobby und Lungenkrebs sind dagegen Peanuts) und die Kosten der Pandemie, gegenüber denen die vor gut 10 Jahren stattgehabte Eurokrise billig erscheint - all dies erledigte ich in meiner persönlichen Rekordzeit von etwa 50 Minuten. Und erhielt langen laustarken Applaus.

Unmittelbar danach war ich von einer Gruppe junger Mütter umringt, deren Stimmen sich nahezu überschlugen: Das hätten sie ja noch nie gehört; warum eigentlich nicht? Ob mir denn nicht bekannt sei, dass in Dänemark die Kinder spätestens mit 8 Jahren ein Smartphone bekommen; oder, dass dänische Schulen voll auf Digitalisierung setzten, etc. Ich versuchte, die Kommentare und Fragen so unaufgeregt wie möglich zu beantworten: Ja, das kann sein; die mit Abstand stärkste Lobby der Welt reagiert auf unangenehme Nachrichten entweder mit Schweigen, dem Erschießen des Übermittlers oder vor allem mit grenzenlosem Hype und Fake-News. War mir nicht bekannt; die deutsche Regierung auch, etc.

Im Laufe des Abends wurde mir klar, dass in Dänemark 3 soziale Experimente laufen. Eines zu einer neuen Form von gemeinschaftlichem Leben, das seit fast 50 Jahren mit bislang ungewissem Ergebnis - und daher offenbar noch immer - läuft. Ein zweites zu einer möglichen neuen Form der Kommunikation von Wissenschaft, von dessen positivem Ausgang ich selbst Gelegenheit hatte, mich zu überzeugen. Und drittens eines zu den Auswirkungen eines neuen digitalen Endgeräts auf die Entwicklung von Kindern und Jugendlichen, von dem ich fürchte, dass es in einem Desaster endet.

\section{Diskussion}

Wie eingangs erwähnt, sind die Dänen das glücklichste Volk der Welt. Sie haben sogar ein eigenes Wort für Glück: Hygge ( $\triangleright$ Abb. 9). Der Bedeutungshof des Wortes ist - wie auch seine Ethymologie - weitaus komplexer als bei „Glück“, bezeichnet „Hygge“ doch auch

- Gemütlichkeit und Behaglichkeit - die Dänen verbrauchen pro Kopf jährlich 6 Kilo Kerzen, doppelt so viele wie Österreich, die Nummer 2 in Europa.

- Gemeinschaft (to hug: umarmen) und Kuscheln mit Verwandten und Freunden - das ganze Wochenende.

- Essen und Trinken - gemeinsam gut (Süßes).

- Eine bestimmte Stimmung (mood) - irgendwo zwischen Glühwein, Trost, Vertrauen - steckt im Wort Hygge, wovon eine von dessen Wurzeln, das altnordische „hugr“ (Stimmung) zeugt.

- Schließlich steckt in Hygge auch die germanische Wurzel „hugjan“ (betrachten, denken), die es auch im Altenglischen als „hycgan“ gibt - es geht also bei Hygge auch um eine Lebenseinstellung, Haltung, Denkungsart - im Bedeutungshof von Präsenz, Harmonie, Schutz, Waffenstillstand, Gemeinschaft, Dankbarkeit und Wohlfühlen.
Dies alles und noch viel mehr entnehme ich dem New York Times Bestseller Hygge, das kein anderer als der Chef des Happiness Research Institute in Kopenhagen, Meik Wiking, geschrieben hat.

Fassen wir zusammen: Was auch immer im Bedeutungshof von Hygge liegt - das Smartphone gehört nicht dazu!

Gemeinsam haben die 3 Experimente, dass der Versuchsplan nicht schriftlich vorliegt, über die zu testenden Hypothesen Unklarheit besteht, und die Auswahl der unabhängigen und abhängigen Variablen noch nicht erfolgt ist. Von größter Bedeutung für unsere Diskussion ist jedoch eine weitere Gemeinsamkeit, nämlich die, dass keine Ethikkommission sie je begutachtet hat. Dann wäre nämlich aufgefallen, dass die Experimente 1 und 2 durchaus mit Hygge vereinbar sind, Experiment 3 jedoch letztlich in nichts anderem besteht, als der Frage nachzugehen, was geschieht, wenn das glücklichste Volk der Welt seine Jugend dem Unglück aussetzt.

Man könnte nun meinen, dass die Dänen ja gerade deswegen, weil sie so glücklich sind, am ehesten ein Experiment zu den Auswirkungen von Unglück wagen könnten. Die Reaktionen der Mütter auf meinen Vortrag sprechen nicht für diese Überlegung. Und selbst wenn sie zutreffend wäre, hätte man die Dänen dennoch vor dem Experiment fragen müssen, ob sie damit einverstanden sind. Sie können sich damit trösten, dass bei den wirklich großen sozialen Experimenten - von der Erfindung des Ackerbaus und der Städte, des Rechts und der Schrift, der Demokratie oder des Kommunismus - die Betroffenen noch nie gefragt wurden. Diese Experimente geschahen einfach, und sie brachten Wohlstand und Not, Leben und Tod - aber bislang keine Erkenntnisse. Denn wer wollte behaupten, dass wir im Hinblick auf die Fragen nach der besten Art, nachhaltig glücklich und gerecht zusammen zu leben, wirklich schlauer geworden wären? Aber immerhin machen die Dänen Experimente. Das zur Wissenschaftskommunikation war erfolgreich und wurde daher institutionalisiert. Die anderen beiden laufen noch.“

\section{Literatur}

[1] Fallesen P, Hind S. Freetown Christiania. Nyt Nordisk Forlag Arnold Busck 2018

[2] Schulze F. Carl Wilhelm von Zehender (1819-1916): Authentische Daten aus seinem Leben. Gesnerus : Swiss Journal of the history of medicine and sciences 1990; 47: 59-66; doi.org/10.5169/seals-521060

[3] Spitzer M. Sex on demand. Satellitennavigation und Geschlechtskrankheiten. Nervenheilkunde 2015; 34: 231-234

[4] Spitzer M. Kurzsichtig wegen mangelnder Weitsicht. Nervenheilkunde 2016; 35: 152-155

[5] Von Zehender CW. Über den Einfluss des Schul-Unterrichts auf Entstehung von Kurzsichtigkeit. Vortrag auf Veranlassung des Vereins-Vorstandes für öffentliche Gesundheitspflege in Rostock am 25. Februar 1880. Nebst einem Anhang enthaltend Entgegnungen von fünfundzwanzig Lehrern des Rostocker Gymnasiums und der Realschule. Stuttgart: Enke; 1880

[6] Wiking M. The little book of hygge. The Danish way to live well. London: Penguin, Random House; 1990 